\title{
Activated ERK1/2 expression in glioblastoma multiforme and in peritumor tissue
}

\author{
GINA LAMA $^{1}$, ANNUNZIATO MANGIOLA ${ }^{2}$, CARMELO ANILE ${ }^{2}$, GIOVANNI SABATINO ${ }^{2}$, \\ PASQUALE DE BONIS ${ }^{2}$, LIBERO LAURIOLA ${ }^{3}$, CECILIA GIANNITELLI ${ }^{1}$, GIUSEPPE LA TORRE ${ }^{4}$, \\ MEENA JHANWAR-UNIYAL ${ }^{5}$, GIGLIOLA SICA ${ }^{1}$ and GIULIO MAIRA ${ }^{2}$ \\ ${ }^{1}$ Institute of Histology and Embryology, ${ }^{2}$ Institute of Neurosurgery, ${ }^{3}$ Institute of Pathology, ${ }^{4}$ Unit of Epidemiology \\ and Biostatistics - Institute of Hygiene, Faculty of Medicine, Catholic University of the Sacred Heart, Rome, Italy; \\ ${ }^{5}$ Departments of Neurosurgery and Pathology, New York Medical College, Valhalla, NY, USA
}

Received January 3, 2007; Accepted February 28, 2007

\begin{abstract}
Anomalies of growth factor signaling have been reported in malignant human gliomas. The extracellular signalregulated kinases (ERKs) play a crucial role in transducing growth factor signals to the nucleus and are involved in a wide range of biological responses, including cell proliferation, differentiation and motility. ERK1/2 is expressed and activated in glioblastoma multiforme (GBM). However, no information is available in literature concerning the presence and activity of ERK $1 / 2$ in the peritumor tissue. In the present study, we evaluated by immunohistochemistry total and phosphorylated ( $t$ and $p$ ) ERK1/2 expression in 31 cases of primary GBM and in tissue surrounding the enhanced lesion at different distances up to $3.5 \mathrm{~cm}$ from the tumor margin. Total ERK1/2 was, as expected, uniformly expressed not only in GBM but in the areas around the tumor also, which showed higher levels of immunolabeling. ERK1/2 activation was observed in GBM as well as in peritumor tissue, with no statistical difference in the level of the enzymatic activities. In particular, in the peritumor tissue pERK1/2 was present independently of neoplastic cells not only in reactive astrocytes, but in apparently normal glial cells also. These results indicate that ERK1/2 pathway may participate in GBM growth and progression. In addition, they strongly suggest that ERK1/2 stimulation may be linked not only to tissue reactivity to tumor invasion, but also to cell motility or represent per se a sign of transformation. Finally, our findings highlight the meaning of the extension of neoplasm fingers beyond the outer margin of GBM. Patients with neoplastic cells at $<10 \%$ or without neoplastic cells in peritumor areas showed a higher survival time compared with those with neoplastic elements at $\geq 10 \%$. In addition, a percentage $\geq 10$ of neoplastic elements in peritumor tissue
\end{abstract}

Correspondence to: Professor Gigliola Sica, Institute of Histology and Embryology, Faculty of Medicine, Catholic University of the Sacred Heart, Largo Francesco Vito 1, I-00168 Rome, Italy

E-mail: ibiis@rm.unicatt.it; gigliola@rm.unicatt.it

Key words: ERK1/2, glioblastoma, peritumor tissue was associated with an approximately 4-fold increased death risk.

\section{Introduction}

Glioblastoma multiforme (GBM) is the most malignant astrocytic tumor, characterized by marked heterogeneity of its cytological composition, high local invasiveness and very low metastatic tendency (1). GBM develops in patients with a mean age of 55 years and the prognosis is extremely poor. The disease is usually treated with aggressive multimodal strategies combining cytoreductive surgery followed by conventional radiation alone or associated with chemotherapy (2). Nevertheless, in spite of all efforts, the median survival of patients is 12 months (3) and the overall 5-year survival rate is approximately $2 \%$ (4).

Studies performed by Burger et al have shown that the distribution of GBM cells cannot be inferred from computerized tomography (CT) images. In fact, while the vast majority of the neoplastic tissue is contained within the contrast-enhancing and peritumoral areas of low-density, the tumor cells in some cases may extend far from this region (5). More recently, Kelly demonstrated by performing $\mathrm{CT}$ and magnetic resonance (MR) imaging-directed stereotactic serial biopsies that in patients harboring both high- and low-grade gliomas tumor cells can be found far from any MR imaging-defined abnormality, at least as far away as $7 \mathrm{~cm}$ (6). On the other hand, Silbergeld and Chicoine reported that cells derived from histologically normal brain obtained from areas at a distance greater than $4 \mathrm{~cm}$ from the edge of GBM (as defined by gross inspection, MR imaging and ultrasonography) could be grown in tissue culture; these cells showed growth rates higher than cells derived from the gross tumor (7).

Interestingly, $>90 \%$ of GBMs recur approximately $2 \mathrm{~cm}$ from the edge of the resection (8). In addition, it has been shown that in patients with GBM the resection of $\geq 98 \%$ of the tumor volume is an independent variable associated with a longer survival time (9).

Alterations of several tumor suppressor genes and oncogenes have been reported to be critical to the initial steps of neoplastic transformation and progression of GBM. In addition, 
several cell cycle regulators and growth factors seem to be involved in the GBM pathogenesis (10). In particular, some growth factors and their receptors, relevant to gliogenesis, are overexpressed in GBM and may establish an autocrine and paracrine loop that supports tumor growth $(11,12)$.

The majority of amplification events in high-grade astrocytoma involves epidermal growth factor receptor (EGF-R). In particular, GBM expresses the endogenous ligands, the epidermal growth factor (EGF) and the transforming growth factor $\alpha$ in addition to EGF-R (12). Approximately $40 \%$ of the GBMs show EGF-R amplification and can express a truncated form of the receptor, constitutively activated, which is due to genomic deletions (13).

Some authors have reported that EGF-R amplification/ overexpression in GBM does not have prognostic relevance $(14,15)$, while others have found it to be a significant predictor of poorer survival in GBM patients and that the EGF-R gene status can be considered a more significant prognostic factor in younger patients ( $<60$ years) (16).

Stimulation of EGF-R in GBM cell lines leads to the increase of secretion of the vascular endothelial growth factor (VEGF) (17). This factor regulates vascular permeability and promotes angiogenesis by inducing proliferation and migration of endothelial cells (18). Moreover, the VEGF receptor flt1 is up-regulated in GBM endothelial cells in vivo and the expression level of the VEGF gene in GBM is elevated and can be increased up to 20- to 50-fold in comparison with low-grade tumors (19).

Signaling pathways involving EGF and other growth factors act via tyrosine kinase receptors or G-protein linked receptors to activate the extracellular signal-regulated kinases 1 and 2 (ERK1/2), proteins belonging to the mitogen-activated protein kinase (MAPK) family. This event is crucial for the activation of several nuclear factors involved in the transcription of genes which regulate cell division, differentiation (20) and migration on extracellular matrix (21).

In normal astrocytes, ERK activation is extremely rare; however, some reports show the presence of activated ERKs as measured by levels of phospho-ERK1/2 (pERK1/2) in astrogliosis, the response of astrocytes to neural injury. The enzymatic activity has been found in brain infarct/ischemia, iatrogenic mechanical/surgical injuries and progressive multifocal leukoencephalopathy. In particular, in the case of mechanical injury, while hypertrophic astrocytes present in the lesions showed pERK1/2 immunoreactivity, only variable subsets of those distant from focal lesions demonstrated ERK activation (22). On the contrary, the ERK1/2 signaling system is present in mature neurons and involved in synaptic plasticity and memory (23). In addition, it has been reported that activated ERK1/2 may contribute to the pathophysiology of depressive disorders in man (24) and may be associated with the emotional component of nociception in rats (25).

More importantly, ERK activation was seen in astrocytic tumors, particularly in GBM. Phosphorylated ERK1/2 expression was also observed in reactive brain near the edge of grade II and III astrocytomas while it was found to be absent in cells distant from the tumor (26). However, the presence of activated ERK1/2 in the peritumor areas of GBM and its relationship to patients' age, gender and survival have never been investigated.
In the present study, we evaluated by means of immunohistochemical procedures the expression of total (t) ERK1/2 and pERK $1 / 2$ in samples obtained from primary GBMs and from the peritumor areas up to $3.5 \mathrm{~cm}$ from the enhanced lesion. Thus, the primary aim of our study was to define the spatial signaling of activated ERK1/2 and to establish if the enzymatic activity may play a role in the progression of GBM. We also attempted to correlate the activated ERK1/2 expression with patients' age and survival.

\section{Patients and methods}

Patients. This study involved 31 adult patients who underwent en bloc surgery for supratentorial GBM at the Institute of Neurosurgery of the Catholic University in Rome between October 2002 and November 2005. On the basis of the patients' clinical history, the GBMs were considered as primary. In fact, the time-interval between first symptom-signs of disease and admission to hospital was very short in all cases and patients had a rapid clinical progression of disease. Moreover, the age-distribution curve of our population fits that of primary GBMs. Nonetheless, we cannot exclude the presence of secondary GBMs with rapid onset-progression of symptoms among our cases.

The clinicopathological characteristics of the patients are reported in Table I. Seventeen patients were male and 14 were female with a mean age of 60.71 years (range 42-72). Thirty-five days after surgery (ranging from 30 to 40 days) patients received external source limited field radiotherapy (with an average dose of $60 \mathrm{~Gy}$ administered in fractions). Simultaneously with radiation therapy, chemotherapy with Temozolomide $\left(\right.$ Temodal $^{\circledR}$ ) was administered at a dose of $75 \mathrm{mg} / \mathrm{m}^{2} /$ day for 7 days per week from the first until the last day of radiotherapy. After a one-month break, patients received up to 6 cycles of Temozolomide at a dose of 150$200 \mathrm{mg} / \mathrm{m}^{2} /$ day according to the standard of 5 days per week every 28 days.

No remarkable adjuvant therapy side effects were noticed. Chemotherapy was discontinued when radiological findings demonstrated a tumor relapse. Patients over 65 years old did not receive a different radio-chemotherapy regimen from younger patients, as we think that a single prognostic factor such as age should not be a reason for undertreatment (27).

Survival time was computed as the time between the operation date and the date of death or 30 June 2006. Of the 31 patients, 6 were still alive, 21 had died of cancer and 4 of other causes. All patients gave consent to use their tumor and peritumor material as well as additional clinical data for research purpose.

Tissue specimens. With the aim of evaluating ERK expression in GBM, extending the analysis to tissue surrounding the enhanced lesion (designated as first area), we obtained tissue samples from a second area (localized at a distance of $<1 \mathrm{~cm}$ from the edge of tumor) and from a third area (localized at a distance starting from $1 \mathrm{~cm}$ up to $3.5 \mathrm{~cm}$ ). Nevertheless, it is important to note that a resection $3.5 \mathrm{~cm}$ far from the enhancing area (i.e. in the apparently healthy white matter) was performed in 2 patients only. Moreover, we did not perform an 'over the enhancing area resection' all around 
Table I. Clinicopathological characteristics of the 31 adult patients with primary GBM (IV WHO) enrolled in the study.

\begin{tabular}{|c|c|c|c|c|c|c|c|}
\hline Patients & Sex & $\begin{array}{c}\text { Age at diagnosis } \\
\text { (years) }\end{array}$ & $\begin{array}{c}\text { Tumor } \\
\text { localization }\end{array}$ & Side & Treatment & $\begin{array}{l}\text { Survival time } \\
\text { (months) }\end{array}$ & $\begin{array}{l}\text { Clinical } \\
\text { outcome }\end{array}$ \\
\hline 1 & M & 69 & Occipital & $\mathrm{L}$ & $\mathrm{CH}+\mathrm{RT}$ & 6 & DOD \\
\hline 2 & M & 67 & Parieto-temporal & $\mathrm{R}$ & $\mathrm{CH}+\mathrm{RT}$ & 8 & DOD \\
\hline 3 & M & 63 & Frontal & $\mathrm{R}$ & $\mathrm{CH}+\mathrm{RT}$ & 11 & DOD \\
\hline 4 & $\mathrm{~F}$ & 47 & Frontal & $\mathrm{L}$ & $\mathrm{CH}+\mathrm{RT}$ & 14 & DOOC \\
\hline 5 & $\mathrm{~F}$ & 65 & Parieto-occipital & $\mathrm{R}$ & $\mathrm{CH}+\mathrm{RT}$ & 18 & DOD \\
\hline 6 & $\mathrm{~F}$ & 58 & Frontal & $\mathrm{R}$ & $\mathrm{CH}+\mathrm{RT}$ & 15 & DOD \\
\hline 7 & $\mathrm{~F}$ & 71 & Frontal & $\mathrm{L}$ & $\mathrm{CH}+\mathrm{RT}$ & 13 & DOD \\
\hline 8 & M & 70 & Temporal & $\mathrm{L}$ & $\mathrm{CH}+\mathrm{RT}$ & 32 & DOD \\
\hline 9 & $\mathrm{~F}$ & 61 & Frontal & $\mathrm{L}$ & $\mathrm{CH}+\mathrm{RT}$ & 12 & DOD \\
\hline 10 & $\mathrm{~F}$ & 68 & Temporal & $\mathrm{L}$ & $\mathrm{CH}+\mathrm{RT}$ & 12 & DOD \\
\hline 11 & M & 58 & Parieto-temporal & $\mathrm{L}$ & $\mathrm{CH}+\mathrm{RT}$ & 19 & DOD \\
\hline 12 & M & 72 & Parietal & $\mathrm{R}$ & $\mathrm{CH}+\mathrm{RT}$ & 12 & DOD \\
\hline 13 & M & 43 & Parietal & $\mathrm{R}$ & $\mathrm{CH}+\mathrm{RT}$ & 19 & DOD \\
\hline 14 & $\mathrm{~F}$ & 62 & Temporal & $\mathrm{R}$ & $\mathrm{CH}+\mathrm{RT}$ & 17 & DOD \\
\hline 15 & $\mathrm{~F}$ & 68 & Frontal & $\mathrm{R}$ & $\mathrm{CH}+\mathrm{RT}$ & 28 & DOD \\
\hline 16 & $\mathrm{~F}$ & 55 & Fronto-temporal & $\mathrm{L}$ & $\mathrm{CH}+\mathrm{RT}$ & 5 & DOD \\
\hline 17 & $\mathrm{~F}$ & 49 & Frontal & $\mathrm{R}$ & $\mathrm{CH}+\mathrm{RT}$ & 28 & Alive \\
\hline 18 & $\mathrm{~F}$ & 47 & Frontal & $\mathrm{L}$ & $\mathrm{CH}+\mathrm{RT}$ & 25 & DOD \\
\hline 19 & $\mathrm{~F}$ & 65 & Frontal & $\mathrm{R}$ & - & 2 & DOOC \\
\hline 20 & M & 69 & Frontal & $\mathrm{L}$ & - & 0.1 & DOOC \\
\hline 21 & M & 72 & Temporal & $\mathrm{R}$ & - & 2 & DOOC \\
\hline 22 & M & 57 & Fronto-parietal & $\mathrm{R}$ & $\mathrm{CH}+\mathrm{RT}$ & 18 & DOD \\
\hline 23 & M & 71 & Parietal & $\mathrm{R}$ & $\mathrm{CH}+\mathrm{RT}$ & 21 & Alive \\
\hline 24 & M & 61 & Fronto-temporal & $\mathrm{R}$ & $\mathrm{CH}+\mathrm{RT}$ & 13 & DOD \\
\hline 25 & $\mathrm{~F}$ & 72 & Parieto-temporal & $\mathrm{R}$ & $\mathrm{CH}+\mathrm{RT}$ & 15 & DOD \\
\hline 26 & $\mathrm{~F}$ & 62 & Fronto-parietal & $\mathrm{R}$ & $\mathrm{CH}+\mathrm{RT}$ & 14 & DOD \\
\hline 27 & M & 68 & Parieto-temporal & $\mathrm{R}$ & $\mathrm{CH}+\mathrm{RT}$ & 12 & DOD \\
\hline 28 & M & 44 & Frontal & $\mathrm{R}$ & $\mathrm{CH}+\mathrm{RT}$ & 18 & Alive \\
\hline 29 & M & 54 & Parieto-occipital & $\mathrm{L}$ & $\mathrm{CH}+\mathrm{RT}$ & 8 & Alive \\
\hline 30 & M & 42 & Temporal & $\mathrm{L}$ & $\mathrm{CH}+\mathrm{RT}$ & 8 & Alive \\
\hline 31 & M & 52 & Parieto-temporal & $\mathrm{R}$ & $\mathrm{CH}+\mathrm{RT}$ & 7 & Alive \\
\hline
\end{tabular}

M, male; F, female; L, left; R, right; DOD, dead of disease; DOOC, dead of other causes.

the lesion, but only towards non-eloquent areas. In all cases where a wide resection was performed, tumor tissues were scanty.

In our series no significant modifications of post-operative KPS were noticed. The anatomical location of the neoplasm did not always allow us to obtain samples in all cases considered: of the 76 specimens of brain tissue 31 were derived from the first area, 26 from the second area and 19 from the third area.

Samples from each zone were immediately fixed in $10 \%$ neutral buffered formalin at room temperature for at least $48 \mathrm{~h}$, washed in water, dehydrated and then embedded in paraffin. All specimens were histologically assessed, using standard hematoxylin and eosin (H\&E) sections, by a single pathologist.
The classification and grading of the tumors were performed according to the criteria of the World Health Organization (WHO) (1). Neoplastic cells were identified by large hyperchromatic and irregular nuclei while reactive astrocytes were recognized by dendritic morphology of their abundant eosinophilic cytoplasm and large, eccentric nuclei, according to Hoelzinger et al (28).

Antibodies. The anti-p44/42 MAPK polyclonal antibody (tERK1/2) and anti-phospho-p44/42 MAPK (Thr202/Tyr204) monoclonal (clone E10) antibody were obtained from Cell Signaling Technology, Inc., Newcastle, USA. Monoclonal antibodies CD68 (clone KP1), and MAC387 (clone MAC387) 
were purchased from Dako Corporation, Carpinteria, CA, USA.

The anti-p44/42 MAPK polyclonal antibody (tERK1/2) detects total level of endogenous p44/42 MAPK (ERK1/2) and the anti-phospho-p44/42 MAPK (pERK1/2) monoclonal antibody specifically detects dually phosphorylated threonine 202 and tyrosine 204 of p44 and p42 MAPK kinases (ERK1 and ERK2).

The biotinylated secondary antibodies (anti-rabbit IgG and anti-mouse $\operatorname{IgG}$ both raised in goat) were from Vector Laboratories, Inc., Burlingame, CA, USA. The specific dually phosphorylated ERK/MAPK peptide (competitor) was a gift from Dr Andreas Nelsbach, Cell Signaling Technology. Specimens of human breast carcinoma and tonsil were used as positive controls for pERK1/2 and both CD68 and MAC387 antibodies, respectively.

Immunohistochemistry. Immunohistochemical detection was carried out on 5- $\mu \mathrm{m}$ thick sections on polylysine-coated slides. The sections were dewaxed in xylene and gradually rehydrated with a series of descending concentrations of ethanol from $100 \%$ to distilled water. Antigen retrieval was performed by microwaving the sections in $10 \mathrm{mmol} / \mathrm{l}$ sodium citrate buffer ( $\mathrm{pH}$ 6.0), with the buffer being brought to boiling point 3 times at $750 \mathrm{~W}$ (10 $\mathrm{min})$; tissue sections in the citrate buffer were allowed to cool at room temperature for at least $20 \mathrm{~min}$.

Quenching of endogenous peroxidase activity was performed in Tris-buffered saline/Triton X-100 (TBST) (50 mmol/l Tris-HCl, $150 \mathrm{mmol} / \mathrm{l} \mathrm{NaCl}, 0,1 \%$ Triton $\mathrm{X}-100, \mathrm{pH} 7.4)$, containing $2 \%$ hydrogen peroxide, for $10 \mathrm{~min}$. Specimens, rinsed first in distilled water and then in TBST, were preincubated in blocking buffer, consisting of 5\% normal goat serum in TBST, for 60 min to block non-specific antibody binding. Incubation with primary antibody (anti-p44/42 MAPK polyclonal antibody or anti-phospho-p44/42 MAPK monoclonal antibody), 1:100 diluted in blocking buffer, was performed overnight at $4{ }^{\circ} \mathrm{C}$. Blocking buffer was also used as diluent for the secondary antibody and for the control peptide. After washing 3 times with TBST, the slides were incubated with biotinylated secondary antibody (goat antirabbit IgG for anti-p44/42 MAPK or goat anti-mouse IgG for anti-phospho-p44/42 MAPK), 1:300 diluted for $60 \mathrm{~min}$, and subsequently with avidin-biotin peroxidase complex (Elite ABC-peroxidase Kit Vector Laboratories). The chromogenic reaction was developed with freshly prepared 3,3'-diaminobenzidine tetrahydrochloride (DAB) solution (Peroxidase DAB Substrate Kit, Vector Laboratories). The slides were lightly counterstained with Mayer's hematoxylin, dehydrated in serial ethyl alcohol solutions, and mounted. Peptide competition controls were performed by preincubating the anti-phosphop44/42 MAPK (Thr202/Tyr204) monoclonal antibody (1 $\mu 1)$ with the phospho-p44/42 MAPK (Thr202/ Tyr204) peptide (5 $\mu \mathrm{l})$, diluted in blocking buffer, at room temperature for $60 \mathrm{~min}$. When primary antibodies were replaced with TBST in negative controls, specific immunoreactivity was not detected.

Two sections of each area were examined by two different observers independently and at least 1,000-2,500 cells from 4 to 10 randomly selected fields in each section were counted. Using a light microscope (Axioskop 2 plus, Zeiss) the percentage of tERK1/2- and pERK 1/2-labeled cells was evaluated at magnification $\mathrm{x} 400$. The few cases with discrepant scoring were re-evaluated jointly on a second occasion and agreement was reached.

Either cytoplasmic and/or nuclear immunoreactivity was considered as positive. The percentage of positive cells was calculated and data were reported as average \pm SD. The percentage of both tERK- and pERK-labeled cells was evaluated considering all immunolabeled cells, apart from the endothelial ones.

The identification of macrophages was obtained by the monoclonal antibodies CD68 (clone KP1, Dako Corp.), 1:400 diluted, and MAC387 (clone MAC387, Dako Corp.), 1:200 diluted. For CD68 staining, heat induced epitope retrieval in sodium citrate buffer ( $\mathrm{pH}$ 6.0) was performed. For MAC387 staining enzymatic predigestion with pronase (bacterial protease type XXIV) was used. Following incubation with antibodies, immunoperoxidase staining was completed using labeled streptavidin-biotin (LSAB kit, Dako Corp.) and diaminobenzidine (DAB, Vector) as chromogen. The nuclei were lightly counterstained with Mayer's hematoxylin.

Statistical analysis. The statistical analyses were carried out using SPSS software (SPSS, Chicago, IL, USA, version 12.0) for Windows. Since the variables considered were not normally distributed, non-parametric tests were applied to find statistically significant differences among groups. Kruskal-Wallis test was used for comparing the levels of tERK1/2 or pERK1/2 expression among the three areas considered. Mann-Whitney test was used for comparing the levels of tERK1/2 or pERK1/2 expression between two groups (unpaired data). Wilcoxon signed rank test was used in order to find a statistically significant difference for paired data (levels of tERK1/2 or pERK1/2 expression for 1 st area vs 2nd area, 1 st area vs 3rd area, 2nd area vs 3rd area).

Patients who did not receive radiotherapy and systemic chemotherapy (3/31) were excluded from the survival analyses (Table I). The diagnostic values of the variables tERK and pERK were assessed by calculating the areas under the receiver operating characteristic (ROC) curves, to establish the best cut-off points to identify the presence of death. The diagnostic accuracy was calculated by sensitivity and specificity.

Patients' survival rates were determined by Kaplan-Meier method and the prognostic significance of potential parameters was determined by univariate analysis (log-rank test and Breslow test). A multivariate analysis was conducted to adjust for possible confounders, using a Cox proportional hazard model. Hazard ratio (HR) and 95\% confidence intervals (CIs) for each variable included in the model were calculated. Stepwise (backward elimination) procedure was used. In all tests probability values of $\mathrm{p}<0.05$ were considered statistically significant.

\section{Results}

The histopathological analysis of all 31 enhanced lesion specimens confirmed the diagnosis of GBM (IV WHO). Typical features of marked hypercellularity, nuclear atypia, frequent mitoses, endovascular proliferation with occasionally glomeruloid structures and sometimes pseudopalisading necrosis were observed (not shown). 
Table II. Percentage of neoplastic cells in peritumor areas of GBM.

\begin{tabular}{cc}
\hline 2nd area & 3rd area \\
$\mathrm{n}=26(\%)$ & $\mathrm{n}=19(\%)$ \\
\hline
\end{tabular}

Neoplastic cells

$\begin{array}{ll}\geq 50 \% & 4 / 26(15.38) \\ \geq 10 \% & 3 / 26(11.54) \\ <10 \% & 6 / 26(23.08)\end{array}$

$2 / 19(10.53)$

$2 / 19(10.53)$

$4 / 19(21.05)$

Absence of neoplastic

$13 / 26(50.00)$

$11 / 19(57.89)$

cells

$\mathrm{n}$, total number of samples.

The peritumor areas were characterized by a decrease in cell density. In these areas, both apparently normal glial cells and reactive hypertrophic astrocytes with large, eccentric, round nuclei were present. Moreover, neoplastic appearing cells, as assessed by large, hyperchromatic and irregular nuclei according to Hoelzinger et al (28), were present in different percentages or completely absent (Table II).

Samples from all three areas were obtained in 14/31 cases. In 12 cases the samples were derived from the first and the second area; in 5 cases the specimens were taken from the first and the third area. Examples of the histology of the different areas stained with $\mathrm{H} \& \mathrm{E}$ are shown in Fig. 1A, D and $\mathrm{G}$.

Expression of tERK1/2 in tumor and peritumor areas. Immunoreactivity for tERK1/2 was detected not only in the tumor but also in the peritumor tissue and it was present in both neoplastic appearing cells and normal or reactive glial cells (Fig. 1B, E and H).

Immunostaining was mostly observed in the cytoplasm where the unphosphorylated ERK1/2 is normally localized; the immunoreaction was less frequent in the nucleus. This would be justified by the fact that the antibody used recognizes the total level of endogenous ERK1/2 independently of the phosphorylation state. The percentage of tERK1/2-positive cells in the first area was quite consistent with $66.3-99.8 \%$ in 29 samples (one sample had $<50 \%$ and another had $0.9 \%$ positive cells). In the second area, the percentage of tERKpositive cells ranged from 73.1 to $100 \%$, and in the third area from 78.2 to $100 \%$ (only one sample displayed $42.4 \%$ positive cells) (Table III).

The level of tERK1/2 expression was higher in the second and in the third area with respect to the first one. In fact, in all 31 cases, the statistical analysis using the Kruskal-Wallis test demonstrated a significant difference in the expression of tERK among the areas $(\mathrm{p}<0.001)$; the mean rank of the first area $(25.33 \%)$ was noticeably lower than those of the second and of the third areas (46.19 and $46.79 \%$, respectively). Similarly, the Mann-Whitney test indicated a significant difference $(p<0.001)$ between tERK expression in the first area (mean rank $21.30 \%$ ) and in the second area (mean
Table III. tERK1/2 expression in the enhanced lesion and in the peritumor areas in 31 cases of GBM.

\begin{tabular}{|c|c|c|c|}
\hline Patient & 1st area & 2nd area & 3rd area \\
\hline 1 & $99.8 \pm 01^{\mathrm{a}}$ & $99.8 \pm 0.3$ & nd \\
\hline 2 & $96.6 \pm 1.6$ & $99.4 \pm 0.2$ & $96.2 \pm 0.4$ \\
\hline 3 & $98.6 \pm 0.2$ & $99.7 \pm 0.0$ & $99.7 \pm 0.2$ \\
\hline 4 & $99.1 \pm 0.6$ & nd & $99.1 \pm 0.8$ \\
\hline 5 & $96.0 \pm 5.2$ & $91.9 \pm 1.1$ & nd \\
\hline 6 & $99.4 \pm 0.2$ & $99.9 \pm 0.1$ & $100.0 \pm 0.0$ \\
\hline 7 & $99.8 \pm 0.7$ & nd & $92.7 \pm 3.9$ \\
\hline 8 & $96.7 \pm 0.6$ & nd & $99.7 \pm 0.0$ \\
\hline 9 & $96.8 \pm 1.3$ & $100.0 \pm 0.0$ & $100.0 \pm 0.0$ \\
\hline 10 & $85.4 \pm 2.1$ & nd & $42.4 \pm 3.0$ \\
\hline 11 & $93.6 \pm 3.0$ & $99.1 \pm 0.4$ & $99.6 \pm 0.1$ \\
\hline 12 & $88.1 \pm 1.1$ & $86.6 \pm 4.0$ & nd \\
\hline 13 & $71.6 \pm 0.8$ & $99.5 \pm 0.4$ & $99.3 \pm 0.4$ \\
\hline 14 & $93.1 \pm 3.6$ & $99.6 \pm 1.1$ & $96.8 \pm 4.4$ \\
\hline 15 & $97.6 \pm 0.1$ & $99.8 \pm 0.3$ & $98.9 \pm 0.8$ \\
\hline 16 & $88.3 \pm 1.2$ & $100.0 \pm 0.0$ & nd \\
\hline 17 & $78.2 \pm 0.0$ & $98.9 \pm 0.2$ & $97.9 \pm 0.2$ \\
\hline 18 & $98.1 \pm 0.5$ & $99.2 \pm 0.3$ & nd \\
\hline 19 & $95.7 \pm 2.9$ & $100.0 \pm 0.0$ & $100.0 \pm 0.0$ \\
\hline 20 & $99.8 \pm 0.2$ & $100.0 \pm 0.0$ & $99.6 \pm 0.0$ \\
\hline 21 & $46.3 \pm 0.5$ & $98.7 \pm 0.2$ & nd \\
\hline 22 & $86.2 \pm 0.6$ & $99.9 \pm 0.1$ & nd \\
\hline 23 & $96.6 \pm 2,7$ & $92.6 \pm 2.1$ & nd \\
\hline 14 & $98.7 \pm 0.1$ & $99.2 \pm 0.7$ & $99.7 \pm 0.1$ \\
\hline 25 & $0.9 \pm 0.1$ & nd & $99.7 \pm 0.0$ \\
\hline 26 & $99.2 \pm 0.1$ & $99.0 \pm 0.4$ & nd \\
\hline 27 & $82.1 \pm 0.1$ & $99.4 \pm 0.6$ & $98.8 \pm 0.3$ \\
\hline 28 & $79.4 \pm 1.0$ & $92.4 \pm 0.7$ & $78.2 \pm 1.1$ \\
\hline 29 & $86.4 \pm 0.9$ & $73.1 \pm 1.2$ & nd \\
\hline 30 & $73.7 \pm 1.3$ & $89.4 \pm 0.3$ & nd \\
\hline 31 & $66.3 \pm 0.1$ & $75.4 \pm 0.8$ & nd \\
\hline
\end{tabular}

aValues represent the mean percentage of positively stained cells for tERK1/2 \pm SD; nd, not determined.

rank $36.81 \%$ ) and between tERK expression in the first area (mean rank 19.53\%) and in the third area (mean rank 33.63\%) $(\mathrm{p}=0.001)$. The Wilcoxon signed rank test also demonstrated a statistically significant difference $(\mathrm{p}=0.003)$ between tERK expression in the first area (median $94.65 \%$ ) and in the second area (median 99.18\%) and between tERK expression in the first area and in the third area (median 99.30\%) $(\mathrm{p}=0.040)$.

These results indicated that the differences are present not only between the areas considered together, but also in the different areas of the same subject. The two latter tests showed no significant difference between the second and the third areas. Moreover, when we analyzed the expression of tERK $1 / 2$ in both the second and the third areas in relation 

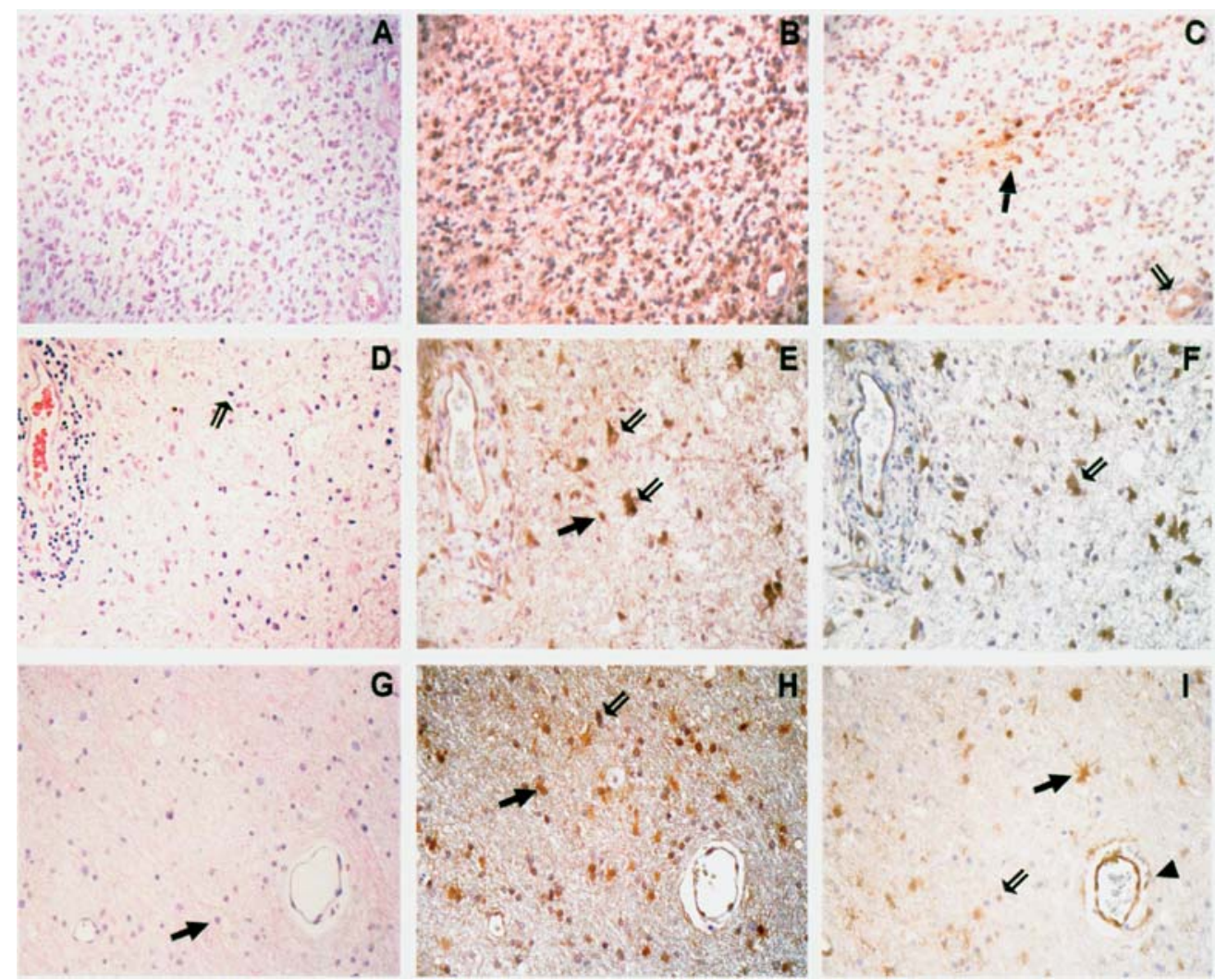

Figure 1. Different patterns of tERK1/2 and pERK1/2 expression in GBM and peritumor tissue. Serial sections from the enhanced lesion (first area, A-C), second (D-F) and third areas (G-I) are shown. A, GBM with high cellularity, cellular anaplasia and vascular proliferation (H\&E staining). B, a high number of tumor cells exhibited tERK immunoreactivity. C, only a limited number of neoplastic cells exhibited nuclear and cytoplasmic immunoreactivity for pERK (black arrow); endothelial cells were positive also (open arrow). D, in a second area characterized by a slightly increased cellularity, some cells with moderately irregular and atypical nuclei, which are suspected for neoplasia were present (open arrow) (H\&E staining). E, tERK immunoreactivity was observed not only in some cells with atypical features (open arrows), but in oligodendroglial (black arrow) and vascular endothelial cells also. F, atypical cells exhibited pERK positivity both in the nucleus and in the cytoplasm (open arrow). G, in an apparently normal peritumor tissue (third area) cells with small round nuclei and scanty cytoplasms were present (black arrow) (H\&E staining). H, tERK immunoreactivity was observed in both a fraction of oligodendrogliallike cells (open arrow) and reactive astrocytes presenting cytoplasmic extensions (black arrow). I, few oligodendroglial-like cells (open arrow) and rare astrocytes (black arrow) exhibited pERK nuclear immunostaining. Also endothelial cells appeared labeled (head arrow). Original magnification: A-I, x200. Hematoxylin counterstain.

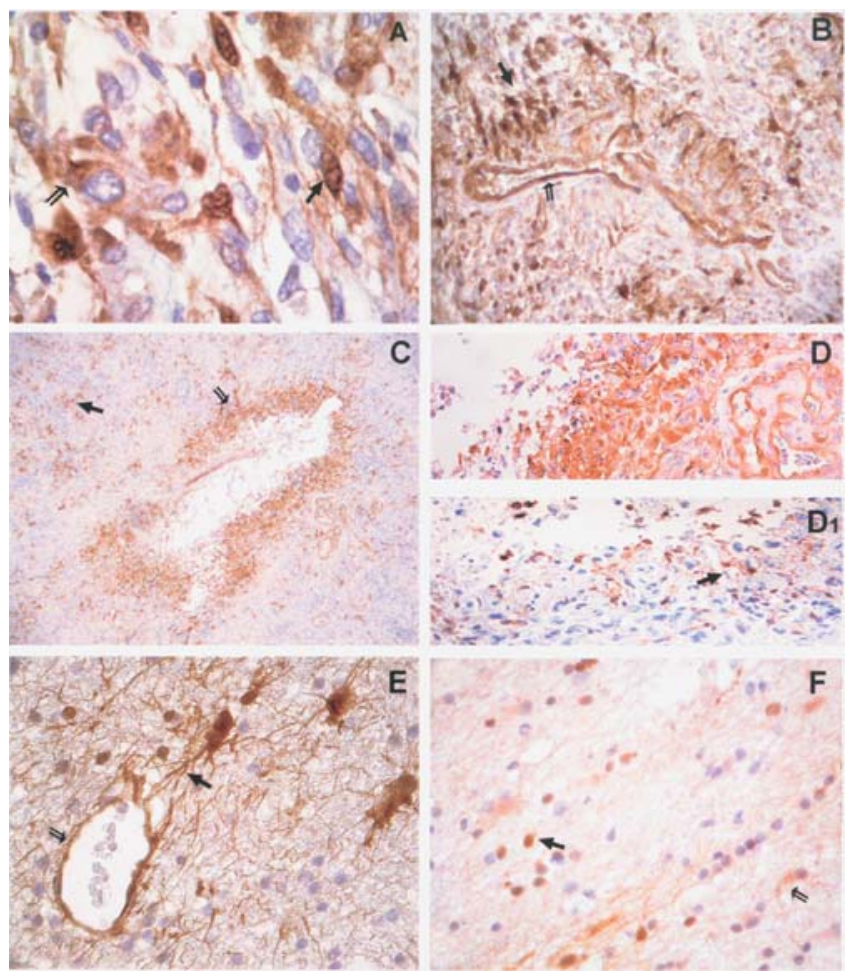

Figure 2. Features of pERK1/2 expression in GBM and peritumor areas. A, a high magnification showing immunostaining in the nucleus (black arrow) as well as in the cytoplasm (open arrow) of some neoplastic cells of the first area. B, increased percentage of immunoreactive cells near a hyperplastic vessel (black arrow) of the first area. Endothelium also expressed pERK1/2 (open arrow). C, enhanced immunoreactivity around a zone of tumor necrosis (open arrow) and an immunoreactive cell cluster (black arrow). $\mathrm{D}$, a high magnification of the cells around the zone of tumor necrosis. D1, immunohistochemistry for Mac387 labeled only a few macrophages, with round or oval morphology (black arrow). E, a reactive astrocyte of the second area showing positive cytoplasmic extensions (black arrow) directed towards a vessel wall in which endothelium (open arrow) is immunostained. F, a third area, in which neoplastic cells were absent, showing apparently normal oligodendroglial cells with nuclear (black arrow) and cytoplasmic (open arrow) immunoreactivity. Original magnification: A, x630; B, x200; C, x50; D, D1, E and F, x400. Hematoxylin counterstain.

to the presence or absence of tumor cells, no statistically significant difference was found $(\mathrm{p}=0.123$ and $\mathrm{p}=0.968$, respectively, Mann-Whitney test) suggesting that neoplastic cells did not influence tERK expression.

Expression of activated ERK1/2 in tumor and peritumor areas. Immunoreactivity for pERK1/2 was detected not only in specimens taken from the tumor but also in the peritumor 
Table IV.pERK1/2 expression in the enhanced lesion and in the peritumor areas in 31 cases of GBM.

\begin{tabular}{|c|c|c|c|}
\hline Patient & 1st area & 2nd area & 3 rd area \\
\hline 1 & $4.6 \pm 0.5^{\mathrm{a}}$ & $5.1 \pm 0.3$ & nd \\
\hline 2 & $11.0 \pm 0.4$ & $5.9 \pm 0.4$ & $22.7 \pm 4.6$ \\
\hline 3 & $2.6 \pm 1.0$ & $23.6 \pm 1.4$ & $3.4 \pm 0.0$ \\
\hline 4 & $1.8 \pm 0.1$ & nd & $7.1 \pm 0.8$ \\
\hline 5 & $28.4 \pm 1.7$ & $8.5 \pm 0.2$ & nd \\
\hline 6 & $87.6 \pm 4.1$ & $10.7 \pm 3.0$ & $16.3 \pm 1.0$ \\
\hline 7 & $19.6 \pm 2.3$ & nd & $89.2 \pm 2.2$ \\
\hline 8 & $32.6 \pm 0.3$ & nd & $16.6 \pm 2.8$ \\
\hline 9 & $34.0 \pm 4.1$ & $18.30 \pm 1.0$ & $38.7 \pm 2.3$ \\
\hline 10 & $12.8 \pm 2.9$ & nd & $6.5 \pm 0.9$ \\
\hline 11 & $22.0 \pm 1.7$ & $26.2 \pm 4.1$ & $34.9 \pm 3.3$ \\
\hline 12 & $16.3 \pm 2.4$ & $22.3 \pm 1.8$ & nd \\
\hline 13 & $32.6 \pm 2.5$ & $48.5 \pm 3.3$ & $34.2 \pm 2.6$ \\
\hline 14 & $63.6 \pm 3.0$ & $22.5 \pm 0.6$ & $20.5 \pm 1.9$ \\
\hline 15 & $25.3 \pm 1.6$ & $32.6 \pm 1.9$ & $43.8 \pm 1.2$ \\
\hline 16 & $16.5 \pm 1.4$ & $34.3 \pm 2.7$ & nd \\
\hline 17 & $21.2 \pm 1.2$ & $35.5 \pm 0.6$ & $25.3 \pm 1.8$ \\
\hline 18 & $54.4 \pm 2.2$ & $46.1 \pm 0.5$ & nd \\
\hline 19 & $35.1 \pm 2.2$ & $14.7 \pm 1.1$ & $29.9 \pm 1.1$ \\
\hline 20 & $81.9 \pm 8.3$ & $96.5 \pm 2.7$ & $90.8 \pm 6.8$ \\
\hline 21 & $43.5 \pm 1.9$ & $43.6 \pm 0.9$ & nd \\
\hline 22 & $38.8 \pm 4.6$ & $90.9 \pm 0.9$ & nd \\
\hline 23 & $9.8 \pm 0.8$ & $18.7 \pm 1.4$ & nd \\
\hline 24 & $35.6 \pm 2.7$ & $48.6 \pm 1.8$ & $18.7 \pm 1.0$ \\
\hline 25 & $0.8 \pm 0.1$ & nd & $5.9 \pm 0.4$ \\
\hline 26 & $28.7 \pm 3.2$ & $43.6 \pm 2.3$ & nd \\
\hline 27 & $17.9 \pm 1.8$ & $41.5 \pm 1.6$ & $70.0 \pm 1.0$ \\
\hline 28 & $18.3 \pm 1.6$ & $9.0 \pm 1.8$ & $17.8 \pm 0.8$ \\
\hline 29 & $41.3 \pm 1.9$ & $51.3 \pm 1.3$ & nd \\
\hline 30 & $4.9 \pm 0.1$ & $14.1 \pm 0.2$ & nd \\
\hline 31 & $54.0 \pm 1.7$ & $53.7 \pm 1.8$ & nd \\
\hline
\end{tabular}

${ }^{a}$ Values represent the mean percentage of positively stained cells for pERK $1 / 2 \pm \mathrm{SD}$; nd, not determined.

tissue (Fig. 1C, F and I). The pERK1/2 staining, in contrast with tERK1/2, was evident in a heterogeneous manner and often seen only in a cell cluster or scattered cells in both enhanced lesion and peritumor areas. The immunoreactivity was mostly localized in the nuclei, whereas cytoplasmic activated ERK1/2 was observed in both the perinuclear region and the cellular processes of glial cells (Fig. 2A and E). In particular, a higher percentage of pERK1/2-positive cells was often observed near hyperplastic vessels (Fig. 2B) as well as in the regions surrounding tumor necrosis (Fig. 2C and D) in the enhanced lesion. The necrotic areas were present in 11 out of the 31 fragments of the enhanced lesion.
Table V. Diagnostic accuracy of pERK and tERK values.

\begin{tabular}{lcccc}
\hline & AUC $(95 \% \mathrm{CI})$ & $\begin{array}{c}\text { Cut-off } \\
\text { value }(\%)\end{array}$ & $\begin{array}{c}\text { Se } \\
(\%)\end{array}$ & $\begin{array}{c}\mathrm{Sp} \\
(\%)\end{array}$ \\
\hline pERK 1st area & $0.508(0.243-0.772)$ & 18.95 & 59.1 & 50.0 \\
tERK 1st area & $0.818(0.638-0.998)$ & 85.83 & 81.8 & 66.7 \\
pERK 2nd area & $0.461(0.169-0.753)$ & 20.54 & 70.6 & 50.0 \\
tERK 2nd area & $0.922(0.812-1.000)$ & 95.60 & 88.2 & 83.3 \\
pERK 3rd area & $0.500(0.225-0.775)$ & 18.23 & 60.0 & 50.0 \\
tERK 3rd area & $0.833(0.616-1.000)$ & 98.39 & 73.3 & 100.0 \\
\hline
\end{tabular}

AUC, area under ROC curve; CI, confidence interval; Se, sensitivity; Sp, specificity.

Around the necrotic areas, cells expressing both CD68 and MAC387 were visualized. The morphology was clearly referable to macrophages, but these cells are only a minor fraction of pERK1/2-labeled cells, as a result of the comparison of pERK and CD68 or MAC387 immunostaining (Fig. 2D1).

In the peritumor tissue, the expression of pERK1/2 was not only limited to the neoplastic cells and reactive astrocytes, but was also present in some apparently normal cells. In fact, the immunolabeling was also found in both the second and third areas when neoplastic cells were absent (Figs. 1I and 2F). Cells characterized by a consistent astrocytic morphology showed pERK1/2 immunoreactivity in fine processes oriented towards the endothelium of the vessel walls (Fig. 2E). Endothelial cells often showed immunoreactivity in the hyperplastic vessels of the enhanced lesion (Figs. 1C and 2B) whereas they were only occasionally immunolabeled in vessels of peripheral areas (Figs. 1I and 2E).

The percentage of pERK-positive cells in the first area ranged from 0.8 to $87.6 \%$, in the second from 5.1 to $96.5 \%$, and in the third from 3.4 to $90.8 \%$ indicating a great variability in the immunoexpression of the protein either in the tumor or in the peritumor regions (Table IV).

There was no significant difference in the pERK1/2 expression between the enhanced lesions and the areas surrounding the neoplasia in the 31 cases considered ( $p>0.1$, for all non-parametric tests). No significant difference existed in the expression of pERK $1 / 2$ between the two peripheral areas $(p=0.476$, Mann-Whitney test; $\mathrm{p}=0.551$, Wilcoxon signed rank test). Moreover, when we analyzed the expression of pERK $1 / 2$ in both the second and the third areas in relation to the presence or absence of tumor cells, no statistically significant difference was found $(p=0.139$ and $p=0.272$, respectively, Mann-Whitney test). Similar statistical results were obtained when the analyses were carried out in the 14 cases in which tissue was obtained from all the three areas (not shown).

ROC curves. The results of the ROC curves, shown in Table V, indicated that the cut-off levels to be utilized in the survival analysis were the following: tERK $=95.60 \%$ for the second area and $\mathrm{tERK}=98.39 \%$ for the third area due to the fact that these cut-offs represent the best compromise between 
sensitivity and specificity. Cut-off levels for pERK and tERK of the first area and pERK of peritumor areas were not considered because the balance between sensitivity and specificity did not reach acceptable values.

Survival analysis of patients and its relationship to gender, age, tumor cells and ERK expression in peritumor areas. Survival analyses of patients in relationship with ERK expression were not performed when the cut-off levels were not identified by ROC curves.

Kaplan-Meier curves, evaluated in the 28 patients who received radio-chemotherapy, indicated that gender, agegroups ( $<65$ and $\geq 65$ years), tERK1/2 of second area (cut-off $=95.60 \%$ ) and tERK1/2 of third area (cut-off $=98.39 \%$ ) were not associated with survival time. Males and females showed a median survival time of 13 and 15 months, respectively (log-rank $=0.08, \mathrm{p}=0.78$; Breslow $=0.01, \mathrm{p}=0.94)$. Age groups showed a median survival time of 17 and 13 months, respectively (log-rank $=0.00, \mathrm{p}=0.97$; Breslow $=0.30, \mathrm{p}=0.58$ ). Patients who had a tERK cut-off level, in the second area, $<95.60 \%$ and $>95.60 \%$ showed a median survival time of 18 and 14 months, respectively (log-rank $=0.94, \mathrm{p}=0.33$; Breslow $=1.09, \mathrm{p}=0.29)$. Patients with a tERK cut-off level, in the third area, $<98.39 \%$ and $>98.39 \%$ showed a median survival time of 13 and 15 months, respectively (log-rank $=0.06, \mathrm{p}=0.81$; Breslow $=0.00, \mathrm{p}=0.96)$.

The percentage of tumor cells ( $\geq 10 \%$ vs $<10 \% /$ absence of tumor cells) in the second and third areas was the only variable associated with survival. Patients with neoplastic cells at $<10 \%$ or without neoplastic cells in both peritumor areas showed a higher survival time compared with those with neoplastic cells at $\geq 10 \%$ (17 vs 12 months; log-rank $=8.39, \mathrm{p}=0.004$; Breslow $=9.08, \mathrm{p}=0.003$ ).

Multivariate analysis using the Cox proportional hazard regression model for the factors such as gender, age-groups, tumor cells in peritumor tissues, and tERK1/2 of the second (cut-off $=95.60 \%$ ) and the third area (cut-off $=98.39 \%$ ) was performed. A stepwise/Wald elimination procedure showed that the only variable associated with patients' death was represented by tumor cells. In particular, the hazard ratio for tumor cells was statistically significant and indicated an $\sim 4$-fold increased death risk associated with a percentage of neoplastic elements of $\geq 10$. Similar results were obtained excluding from the analysis tERK $1 / 2$ of the second and third area $(\mathrm{HR}=3.68$; $95 \%$ CI $1.38-9.79 ; \mathrm{p}=0.009)$.

\section{Discussion}

In this study, we reported the presence of neoplastic cells in the peritumor tissue of GBM (up to $3.5 \mathrm{~cm}$ from the tumor margin), in agreement with results obtained by other authors (7). Moreover, we demonstrated that a high percentage of neoplastic cells in the peritumor areas is associated with survival, indicating the hazard ratio for this variable to be an approximately 4-fold increase of death risk. Interestingly, for the first time, activated ERK1/2 was shown to be present not only in the enhanced lesion but also in the tissue surrounding the neoplasia both in reactive astrocytes and in apparently normal cells, even in the absence of neoplastic elements. It is worth mentioning that the level of pERK1/2 expression did not differ significantly between the enhanced lesion and the peritumor areas.

Aberrant signal transduction pathways are involved in glioblastoma pathogenesis and progression (29). ERK1/2 is a crucial component of the pathways activated by growth factors such as EGF, platelet derived growth factor, insulin-like growth factor, brain-derived neurotrophic factor and fibroblast growth factor (30-32).

It has been shown that translocation of pERK $1 / 2$ in the nucleus and subsequent phosphorylation of nuclear targets is required for the expression of mitogen-induced genes and reentry in the cell cycle (33). Depending on the cellular context, phosphorylation of ERK1/2 leads to either cell proliferation or differentiation (34), but constitutive activation is sufficient to promote transformation in mammalian cells (35).

ERK activation may be linked to the expression of cyclin D1, a protein known to mediate G1 progression following a mitogenic stimulus (36). It is interesting to note that cyclin D1 expression is highly variable in GBM (37) and that, according to this, we found a high intratumoral and intertumoral variability in pERK $1 / 2$ immunolabeling. The same variability of $\mathrm{pERK} 1 / 2$ expression was found in the peritumor areas and this determined a lack of statistical difference in the expression between the enhanced lesion and peritumor tissue. In agreement with Mandell et al (26), we observed a spatial pattern of ERK activation. In fact, elevated pERK1/2 expression was found in cells surrounding microvasculature and necrotic areas. This may be due to factors produced by microvasculature cells and by dying tumor cells or macrophages, even if it is worth mentioning that phagocytes represent only a minor fraction of the immunolabeled cells.

Immunoreactivity for pERK $1 / 2$ in the tissue surrounding the neoplasia was not limited to tumor cells but it was also seen in apparently normal glial cells. Consistent with findings from other authors, we observed pERK1/2 expression in reactive astrocytes (26).

It appears that ERK1/2 activation is deeply involved in the maintenance of reactive astrocytic phenotype due to a chronic activation of this signaling pathway related to either an altered expression of some components, that render the activation independent from extracellular stimuli, or to the continuous presence of external stimulatory factors (22).

The activation of ERK1/2 pathway in apparently normal glial cells might equally be linked to continuous stimuli by tumor-derived growth factors, perhaps from the neighbouring neoplastic or pre-neoplastic cells or via the extracellular matrix. Its linkage to the acquisition of neoplastic phenotype cannot be excluded, even if this activation may not represent the only way by which transformation occurs.

Based on findings by Silbergeld and Chicoine, who demonstrated that cultured cell lines, obtained from apparently normal brain tissue, were a subpopulation of cells derived from the tumor showing a similar motility but a higher growth rate than tumor cells (7), we hypothesized that apparently normal cells expressing pERK1/2 in peripheral areas might harbor a 'transformed phenotype'.

The neighboring tissues of GBM, where tumor re-growth is observed in $90 \%$ of cases, may represent a proper site to study the complex phenomenon of tumor progression. The presence of cytoplasmic activated ERK1/2, also in apparently 
normal cells located in peripheral areas, suggests possible non-transcriptional roles, such as the regulation of cytoarchitecture and cell migration (26). Klemke et al showed that activated ERK1/2 can directly phosphorylate myosin light chain kinase, a key regulator of both cell motility and contraction, thus increasing its ability to phosphorylate myosin light chains, which, in turn, promotes the cytoskeletal contraction necessary for cell motility (21). Moreover, persistent activation of ERK $1 / 2$ or prolonged retention of pERK $1 / 2$ in the nucleus has been shown to induce cell motility $(38,39)$. These observations indicate that pERK $1 / 2$ can also affect the invasive process by directly activating the intracellular motility mechanisms.

GBM progression is also linked to extracellular matrix protein degradation. With regard to this, Lakka et al showed that production of MMP-9, a collagenase involved in basement membrane dissolution and in the invasive behavior of the human glioblastoma cell line SNB19, is regulated by ERKdependent signaling modules. Moreover, interfering with this pathway reduced cell invasiveness (40).

We could not exclude that ERK activation observed in the GBM peritumor tissue may be linked at the same time to cell division and migration. It is generally believed that cells cannot move and divide at the same time, the so-called 'go or grow' hypothesis. Nevertheless, Angers-Loustau et al demonstrated that the Src family kinase-specific pharmacologic inhibitors PP2 and SU6656 significantly inhibited both the invasion and the growth of the human glioblastoma cell line U251 in a three-dimensional collagen type I matrix (41). We were not able to establish a correlation between ERK activation (in both enhanced lesion and peritumor tissue) and survival, probably due to the small group of patients entered into the study and the great variability of the data.

On the contrary, Mawrin et al reported that the MAP kinase pathway correlates with proliferation in GBM and that a patient subgroup exists, where expression of MAP kinaserelated signaling proteins is associated with poorer prognosis (42). More recently, another study showed that elevated expression of pERK is associated with a poor response to radiotherapy and represents an independent prognostic factor in GBM (43). ERK1/2 activation in peritumor tissue is, in our opinion, meaningful. Its presence is not strictly linked to neoplastic cells, which both dividing and migrating determine per se an increased death risk.

In addition, in our experience, apart from activated astrocytes, apparently normal cells do express pERK1/2. This expression might indicate that, in some way, they share with both neoplastic and reactive glial cells a state in which the ERK1/2 pattern is functioning.

On the basis of the fact that normal glial cells do not show ERK activation, we can not exclude that the immunolabeling of these elements may be a sign of transformation. This hypothesis is supported by the finding that the peritumor tissue is inevitably destined to become the area of tumoral invasion.

The finding that tERK1/2 is uniformly expressed in both the tumor and peritumor tissue is in complete agreement with data from Mandell et al (26) concerning the uniform presence of tERK1/2 in non-neoplastic gray and white matter and in tumor and peritumoral white matter. On the other hand, in some cases ERK1/2 activation was low in the second and third areas and this is in complete accordance with data reported in some diseases. In fact, it has been shown that, in epilepsy/hippocampal sclerosis, the immunostaining patterns observed for pERK $1 / 2$ do not merely reflect the relative abundance of tERK1/2 protein (22).

In our experience, the expression of tERK $1 / 2$ was a little higher in the peripheral areas compared with the enhanced lesion, which is difficult to understand from the biological point of view. The presence of tERK $1 / 2$ in the majority of cells indicates that it could be potentially activated in relationship with division, migration and survival.

Although the regulation of ERK $1 / 2$ signalling cascade is only partially understood, some compounds that target this pathway have already entered clinical practice (44). Our findings clearly suggest that a better knowledge of ERK1/2 function in GBM may represent the most appropriate scientific basis to assess targeted therapies and their application timing.

\section{Acknowledgments}

This study was supported by grants from MIUR (FIRB: RBNE01MBEC) and from Atena Onlus, as well as by financial research contributions from the Catholic University of the Sacred Heart. We thank Mrs. Sandra De Silvestri for her technical assistance.

\section{References}

1. Kleihues P, Burger PC, Collins VP, Newcomb EW, Ohgaki H and Cavenee WK: Glioblastoma. In: Pathology and Genetics of Tumours of the Nervous System. Kleihues P and Cavenee WK (eds). IARC Press, Lyon, pp29-39, 2000.

2. Stupp R, Masori WP, van den Bent MJ, et al: Radiotherapy plus concomitant and adjuvant temozolomide for glioblastoma. N Engl J Med 352: 987-996, 2005.

3 Behin A, Hoang-Xuan K, Carpentier AF and Delattre JY: Primary brain tumours in adults. Lancet 361: 323-331, 2003

4. McLendon RE and Halperin EC: Is the long-term survival of patients with intracranial glioblastoma multiforme overstated? Cancer 98: 1745-1748, 2003.

5. Burger PC, Heinz ER, Shibata T and Kleihues P: Topographic anatomy and CT correlations in the untreated glioblastoma multiforme. J Neurosurg 68: 698-704, 1988.

6. Kelly PJ: Technology in the resection of gliomas and the definition of madness. J Neurosurg 101: 284-286, 2004.

7. Silbergeld DL and Chicoine MR: Isolation and characterization of human malignant glioma cells from histologically normal brain. J Neurosurg 86: 525-531, 1997.

8. Hochberg FH and Pruitt A: Assumptions in the radiotherapy of glioblastoma. Neurology 30: 907-911, 1980.

9. Lacroix M, Abi-Said D, Fourney DR, et al: A multivariate analysis of 416 patients with glioblastoma multiforme: prognosis, extent of resection, and survival. J Neurosurg 95: 190-198, 2001.

10. Boudreau CR, Yang I and Liau LM: Gliomas: advances in molecular analysis and characterization. Surg Neurol 64: 286-294, 2005.

11. Hermanson M, Funa K, Hartman M, Claesson-Welsh L, Heldin CH, Westermark B and Nister M: Platelet-derived growth factor and its receptors in human glioma tissue: expression of messenger RNA and protein suggests the presence of autocrine and paracrine loops. Cancer Res 52: 3213-3219, 1992.

12. Ekstrand AJ, James CD, Cavenee WK, Seliger B, Pettersson RF and Collins VP: Genes for epidermal growth factor receptor, transforming growth factor alpha, and epidermal growth factor and their expression in human gliomas in vivo. Cancer Res 51: 2164-2172, 1991

13. Wong AJ, Ruppert JM, Bigner SH, Grzeschik CH, Humphrey PA, Bigner DS and Vogelstein B: Structural alterations of the epidermal growth factor receptor gene in human gliomas. Proc Natl Acad Sci USA 89: 2965-2969, 1992. 
14. Quan AL, Barnett GH, Lee SY, et al: Epidermal growth factor receptor amplification does not have prognostic significance in patients with glioblastoma multiforme. Int J Radiat Oncol Biol Phys 63: 695-703, 2005.

15. Chakravarti A, Seiferheld W, Tu X, et al: Immunohistochemically determined total epidermal growth factor receptor levels not of prognostic value in newly diagnosed glioblastoma multiforme: report from the Radiation Therapy Oncology Group. Int J Radiat Oncol Biol Phys 62: 318-327, 2005.

16. Shinojima N, Tada K, Shiraishi S, et al: Prognostic value of epidermal growth factor receptor in patients with glioblastoma multiforme. Cancer Res 63: 6962-6970, 2003.

17. Goldman CK, Kim J, Wong WL, King V, Brock T and Gillespie GY: Epidermal growth factor stimulates vascular endothelial growth factor production by human malignant glioma cells: a model of glioblastoma multiforme pathophysiology. Mol Biol Cell 4: 121-133, 1993.

18. Maher EA, Furnari FB, Bachoo RM, Rowitch DH, Louis DN, Cavenee WK and De Pinho RA: Malignant glioma: genetics and biology of a grave matter. Genes Dev 15: 1311-1333, 2001.

19. Weindel K, Moringlane JR, Marmé D and Weich HA: Detection and quantification of vascular endothelial growth factor/vascular permeability factor in brain tumor tissue and cyst fluid: the key to angiogenesis? Neurosurgery 35: 439-449, 1994.

20. Marshall CJ: Specificity of receptor tyrosine kinase signaling: transient versus sustained extracellular signal-regulated kinase activation. Cell 80: 179-185, 1995.

21. Klemke RL, Cai S, Giannini AL, Gallagher PJ, De Lanerolle P and Cheresh DA: Regulation of cell motility by mitogen-activated protein kinase. J Cell Biol 137: 481-492, 1997.

22. Mandell JW and VandenBerg SR: ERK/MAP kinase is chronically activated in human reactive astrocytes. Neuroreport 10: 3567-3572, 1999.

23. Sweatt JD: The neuronal MAP kinase cascade: a biochemical signal integration system subserving synaptic plasticity and memory. J Neurochem 76: 1-10, 2001.

24. Dwivedi Y, Rizavi HS, Roberts RC, Conley RC, Tamminga CA and Pandey GN: Reduced activation and expression of ERK1/2 MAP kinase in the post mortem brain of depressed suicide subjects. J Neurochem 77: 916-928, 2001.

25. Gioia M, Galbiati S, Rigamonti L, Moscheni C and Gagliano N: Extracellular signal-regulated kinases 1 and 2 phophorylated neurons in the tele- and diencephalon of rat after visceral pain stimulation: an immunocytochemical study. Neurosci Lett 308: 177-180, 2001

26. Mandell JW, Hussaini IM, Zecevic M, Weber MJ and Vanden Berg SR: In situ visualization of intratumor growth factor signaling. Immunohistochemical localization of activated ERK/MAP kinase in glial neoplasms. Am J Pathol 153: 14111423,1998

27. Mangiola A, Maira G, De Bonis P, Porso M, Pettorini B, Sabatino $G$ and Anile C: Glioblastoma multiforme in the elderly: a therapeutic challenge. J Neurooncol 76: 159-163, 2006.

28. Hoelzinger DB, Mariani L, Weis J, et al: Gene expression profile of glioblastoma multiforme invasive phenotype points to new therapeutic targets. Neoplasia 7: 7-16, 2005.
29. Kapoor GS and O'Rourke DM: Mitogenic signaling cascades in glial tumors. Neurosurgery 52: 1425-1435, 2003.

30. Tournier C, Pomerance M, Gavaret JM and Pierre M: MAP kinase cascade in astrocytes. Glia 10: 81-88, 1994.

31. Roback JD, Marsh HN, Downen M, Palfrey HC and Wainer BH: BDNF-activated signal transduction in rat cortical glial cells. Eur J Neurosci 7: 849-862, 1995

32. Leirdal $\mathrm{M}$ and Sioud $\mathrm{M}$ : Protein kinase $\mathrm{C}$ alpha isoform regulates the activation of the MAP kinase ERK1/2 in human glioma cells: involvement in cell survival and gene expression. Mol Cell Biol Res Commun 4: 106-110, 2000.

33. Brunet A, Roux D, Lenormand P, Dowd S, Keyse S and Pouysségur J: Nuclear translocation of p42/p44 mitogen-activated protein kinase is required for growth factor-induced gene expression and cell cycle entry. EMBO J 18: 664-674, 1999.

34. Cowley S, Paterson H, Kemp P and Marshall CJ: Activation of MAP kinase kinase is necessary and sufficient for PC12 differentiation and for transformation of NIH 3T3 cells. Cell 77: 841-852, 1994

35. Mansour SJ, Matten WT, Hermann AS, et al: Transformation of mammalian cells by constitutively active MAP kinase kinase. Science 265: 966-970, 1994.

36. Weber JD, Raben DM, Phillips PJ and Baldassare JJ: Sustained activation of extracellular-signal-regulated kinase 1 (ERK1) is required for the continued expression of cyclin D1 in G1 phase. Biochem J 326: 61-68, 1997.

37. Fiano V, Ghimenti C, Imarisio S, Silengo L and Schiffer D pAkt, cyclin D1 and p27/Kip.1 in glioblastomas with and without EGFR amplification and PTEN mutation. Anticancer Res 24: 2643-2647,2004.

38. Krueger JS, Keshamouni VG, Atanaskova N and Reddy KB Temporal and quantitative regulation of mitogen-activated protein kinase (MAPK) modulates cell motility and invasion. Oncogene 20: 4209-4218, 2001.

39. Tanimura S, Nomura K, Ozaki K, Tsujimoto M, Kondo T and Kohno M: Prolonged nuclear retention of activated extracellular signal-regulated kinases $1 / 2$ is required for hepatocyte growth factor-induced cell motility. J Biol Chem 277: 28256-28264, 2002.

40. Lakka SS, Jasti SL, Kyritsis AP, Yung WKA, Ali-Osman F, Nicolson GL and Rao JS: Regulation of MMP-9 (type IV collagenase) production and invasiveness in gliomas by the extracellular signal-regulated kinase and jun amino-terminal kinase signaling cascades. Clin Exp Metastasis 18: 245-252, 2000

41. Angers-Loustau A, Hering R, Werbowetski TE, Kaplan DR and Del Maestro RF: SRC regulates actin dynamics and invasion of malignant glial cells in three dimensions. Mol Cancer Res 2: 595-605, 2004.

42. Mawrin C, Diete S, Treuheit T, et al: Prognostic relevance of MAPK expression in glioblastoma multiforme. Int $\mathbf{J}$ Oncol 23: 641-648, 2003

43. Pelloski CE, Lin E, Zhang Li, et al: Prognostic associations of activated mitogen-activated protein kinase and Akt pathways in glioblastoma. Clin Cancer Res 12: 3935-3941, 2006.

44. Mischel PS and Cloughesy TF: Targeted molecular therapy of GBM. Brain Pathol 13: 52-61, 2003. 